\title{
A two-stage stochastic model for distribution logistics with transshipment and backordering: stochastic vs deterministic solutions
}

\author{
Rossana Cavagnini, Luca Bertazzi and Francesca Maggioni
}

\begin{abstract}
We present a two-stage stochastic program for a distribution logistic system with transshipment and backordering under stochastic demand and we first argue that it is NP-hard. Then, we perform a computational analysis based on a distribution network. In the case with two retailers, we show that modeling uncertainty with a stochastic program leads to better solutions with respect to the ones provided by the deterministic program, especially if limited recourse actions are admitted. Although there are special cases in which the deterministic and the stochastic solutions select the same retailers towards which sending items, in general, the deterministic solution cannot be upgraded in order to find the optimal solution of the stochastic program. Finally, in the case with four retailers, transshipment can provide more flexibility and better results.
\end{abstract}

Keywords: Optimization under Uncertainty, Transshipment, Backordering, Stochastic solution analysis

\section{Introduction}

In recent years, competition pressure has increased and logistics has become more and more crucial for the success of companies due to its impact on costs and service levels. An efficient distribution system is fundamental to satisfy customers' requests with reduced lead times and with a good service level. Traditionally, the distribution network is organized as a hierarchical process in which the flow of goods is shipped

Rossana Cavagnini

University of Bergamo, Via dei Caniana, 2, Bergamo, Italy, e-mail: r.cavagnini@studenti.unibg.it

Luca Bertazzi

University of Brescia, Contrada Santa Chiara, 50, Brescia, Italy, e-mail: luca.bertazzi@unibs.it

Francesca Maggioni

University of Bergamo, Via dei Caniana, 2, Bergamo, Italy, e-mail: francesca.maggioni@unibg.it 
from the uppermost level of the distribution chain to the lowest. One of the purposes of this paper is to study a more flexible distribution network, where the shipment of products between locations at the same level of the distribution system is admitted. This strategy is called transshipment and it allows companies to reduce stock out risks, to share surplus stocks and to improve warehouses management, coping with demand uncertainty.

Based on the inventory system, ordering and transshipment characteristics, [12] present a complete review of the transshipment literature. Examples of stochastic transshipment problems are [5], where fixed replenishment costs are taken into account, while [11] considers the unidirectional transshipment problem, where locations have different backordering and stockout costs. Backordering is not considered in [15], while [16] studies the multi-location transshipment problem including lead times. Finally, [14] proposes a stochastic transshipment model for humanitarian emergencies.

Our contribution is to provide insights about the importance of considering uncertainty in a distribution system with transshipment and backordering.

The remainder of the paper is organized as follows. Sect. 2 presents the problem description and formulation. Sect. 3 shows our computational results and, finally, in Sect. 4 , conclusions and research perspectives are outlined.

\section{Problem Description and Formulation}

The analyzed problem deals with a single echelon distribution system composed of a single supplier and a set $\mathscr{I}$ of $M$ retailers with a centralized decision making. Transshipment is admitted and, in order to keep track of the origin and destination of product flows, we represent retailers performing transshipment by index $i$ and retailers receiving transshipped quantities by index $j(i \in \mathscr{I}, j \in \mathscr{I})$. In this problem transshipment is intra-level (since it involves only retailers), bi-directional (each retailer can both transship products to other retailers and receive products from them) and reactive (it is performed in emergency situations, after demand realization). We deal with a single product complete pooling transshipment (retailer $i$ can not keep any inventory quantity if retailer $j$ has a shortage of product), where the priority principle is respected (each retailer satisfies its demand at first and then transshipment is performed if necessary), backordering to supplier is allowed and, consequently, the demand can potentially be covered with supplied quantities, with transshipment quantities and with backordered quantities. The unsatisfied demand represents a lost sale. Since retailers are supposed to be close to each other, lead times are considered negligible. Our problem is described on two time intervals: $t_{0}$, which represents the time at which we have to take the decision about the quantities to ship from the supplier to retailers and $t_{1}$, in which, after demand realization, we decide the quantities to transship and the quantities to backorder.

Moreover, the problem is characterized by risk presence: the demand is a phenomenon which can not be exactly forecast, but it is stochastic. We denote by $d$ all 
possible values for the demand, that is a random variable having discrete (mutually indipendent) probability distributions $\mathscr{D}_{i}$, defined over the support $\mathscr{U}_{1}=\{\underline{d}, \ldots, \bar{d}\}$, where $0<\underline{d} \leq \bar{d}$. Furthermore, we represent by $\mathscr{S}$ the set of scenarios $s, s=1, \ldots, S$ and by $p r^{s}$ the probability of each scenario $s \in \mathscr{S}$, so that $d_{i}^{s}$ denotes the demand realization for retailer $i$ in scenario $s$. The measure adopted to evaluate the system performance is the total expected cost.

At time $t_{0}$, the decision variables of this model are $x_{i}$, which represent the decisions to take at the first stage, i.e. the quantity to ship from the supplier to each retailer $i$, taking into account the supplier's total inventory availability $q$ and the associated unit inventory cost $h_{0}$. We introduce a capacity $C_{i}$ for each vehicle employed in the shipment of units from the supplier to retailer $i$ and an integer variable $v_{i}$, standing for the number of total vehicles used to serve retailer $i$ by direct shipping. The transportation cost between the supplier and each retailer is represented by a variable cost $f_{i}$, proportional to the number of shipped units and by a fixed component $F_{i}$, paid for each vehicle used.

If retailer $j$ has to face a demand $d_{j}^{s}$ greater than the initial inventory level $\bar{I}_{i 0}$ plus the quantity $x_{i}$ received from the supplier, transshipment and/or backordering can be used to avoid stock-out. Thus, at $t_{1}$ the decision variables are represented by $y_{i j}^{s}$ which stand for the quantity to transship from retailer $i$ to retailer $j$, for each possible scenario $s$, after the demand realization $d_{i}^{s}$ and by $b_{i}^{s}$ which represent the quantity to backorder from the supplier for each retailer and for each possible scenario $s$, after demand realization $d_{i}^{s}$. On one hand, we introduce a capacity $C^{T}$ for vehicles used to transship units (note that the capacity of vehicles used to ship units from supplier to retailers is typically bigger than the capacity of vehicles used for transshipment) and integer variables $V_{i j}^{s}$ representing the number of vehicles employed for transshipment from retailer $i$ to retailer $j$ for each scenario $s$. The total transshipment cost is composed of a unit cost $t_{i j}$ for each transshipped unit and a fixed cost $T_{i j}$ for each vehicle used. On the other hand, backordering is done by using vehicles with the same capacity $C_{i}$ of vehicles used for the shipment from the supplier to retailer $i$ and we represent the number of vehicles used for backordering with the variables $r_{i}^{s}$. The total backordering cost is composed of a unit backordering cost $g_{i}$ for each backordered unit and a fixed cost $G_{i}$ for each vehicle used. Finally, the variables $I_{i}^{S}$ represent the balance quantity at each retailer $i$ for each scenario $s$ and they are given by the sum of the initial inventory level $\bar{I}_{i 0}$ plus the quantity received from the supplier, the quantity received through transshipment and through backordering minus the sum of the customers' demand and of the transshipped units. If this quantity is positive, it stands for the inventory level and the associated unit cost is represented by $h_{i}$. If the quantity is negative, then the balance quantity stands for the stock-out quantity and retailer $j$ has to pay a unit penality cost $p_{j}$. In particular, if the product surplus at retailer $i$ is transshipped to retailer $j$, but it is not sufficient to fully cover the shortage of product of retailer $j$, and no quantities are backordered, retailer $i$ has neither inventory nor stock-out costs, while retailer $j$ has to face stock-out costs for the unsatisfied demand. We also consider the warehouse capacity $Q_{i}$ for each retailer $i$. 
Consequently, we formulate the following integer non linear two stage stochastic programming model.

$$
\begin{aligned}
& \text { Model } \mathscr{T} \\
& \min h_{0}\left(q-\sum_{i \in \mathscr{I}} x_{i}\right)+\sum_{i \in \mathscr{I}}\left(f_{i} x_{i}+F_{i} v_{i}\right)+ \\
& +\sum_{s \in \mathscr{S}} p r^{s}\left[h_{0}\left(q-\sum_{i \in \mathscr{I}} x_{i}-\sum_{i \in \mathscr{I}} b_{i}^{s}\right)+\sum_{i \in \mathscr{I}}\left(g_{i} b_{i}^{s}+G_{i} r_{i}^{s}\right)+\right. \\
& \left.+\sum_{i \in \mathscr{I}} h_{i} \max \left\{I_{i}^{S}, 0\right\}+\sum_{i \in \mathscr{I}} \sum_{j \in \mathscr{I}: i \neq j}\left(t_{i j} y_{i j}^{s}+T_{i j} V_{i j}^{s}\right)-\sum_{j \in \mathscr{I}} p_{j} \min \left\{I_{j}^{s}, 0\right\}\right]
\end{aligned}
$$

s.t.

$$
\begin{gathered}
\sum_{i \in \mathscr{I}}\left(x_{i}+b_{i}^{s}\right) \leq q \quad s \in \mathscr{S} \\
I_{i}^{s}=\bar{I}_{i 0}+x_{i}+b_{i}^{s}-d_{i}^{s}+\sum_{j \in \mathscr{I}: i \neq j}\left(y_{j i}^{s}-y_{i j}^{s}\right) \quad i \in \mathscr{I}, s \in \mathscr{S} \\
I_{i}^{s} \leq Q_{i} \quad i \in \mathscr{I}, s \in \mathscr{S} \\
x_{i} \leq C_{i} v_{i} \quad i \in \mathscr{I} \\
b_{i}^{s} \leq C_{i} r_{i}^{s} \quad i \in \mathscr{I}, s \in \mathscr{S} \\
y_{i j}^{s} \leq C^{T} V_{i j}^{s} \quad i \in \mathscr{I}, j \in \mathscr{I}: j \neq i, s \in \mathscr{S} \\
x_{i} \geq 0 \text { integer } \quad i \in \mathscr{I} \\
y_{i j}^{s} \geq 0 \text { integer } \quad i \in \mathscr{I}, j \in \mathscr{I}: j \neq i, s \in \mathscr{S} \\
b_{i}^{s} \geq 0 \text { integer } \quad i \in \mathscr{I}, s \in \mathscr{S} \\
v_{i} \geq 0 \text { integer } \quad i \in \mathscr{I} \\
r_{i}^{s} \geq 0 \text { integer } \quad i \in \mathscr{I}, s \in \mathscr{S} \\
V_{i j}^{s} \geq 0 \text { integer } \quad i \in \mathscr{I}, j \in \mathscr{I}: j \neq i, s \in \mathscr{S} \\
I_{i}^{s} \text { free } \quad i \in \mathscr{I}, s \in \mathscr{S}
\end{gathered}
$$

where the objective function (1) represents the minimization of the total expected cost, obtained through the sum of the supplier's inventory cost, the total shipment costs from supplier to retailers, the expected supplier's inventory costs, the total expected backordering cost, the total expected retailers' inventory cost, the total expected transshipment costs and the expected stock-out costs. Constraints (2) implies that the total quantity shipped from the supplier to all retailers (through usual shipment and backordering) cannot be greater than the supplier's initial inventory. Constraints (3) are the balance constraints. Constraints (4) imply that the balance quantity (computed as in (3)) cannot exceed the warehouse capacity $Q_{i}$ for each retailer $i$. Constraints (5), (6) and (7) link together the decision variables $x_{i}, b_{i}^{s}$ and $y_{i j}^{s}$ with the respective integer variables $v_{i}, r_{i}^{S}$ and $V_{i j}^{s}$ so that if the first ones are positive, these quantities are splitted in a certain number of vehicles represented by the latter ones, considering the respective vehicles capacities $C_{i}$ and $C^{T}$ and, consequently, the 
associated fixed costs $F_{i}, G_{i}$ and $T_{i j}$ are charged in the objective function. Finally, constraints from (8) to (14) are variables definition constraints. Due to the nonlinearity of Model $\mathscr{T}$, we linearize it following the approach described in [4] and we call the linearized problem "Model $\mathscr{T}^{\mathscr{L}}$ ". Finally, we notice that Model $\mathscr{T}^{\mathscr{L}}$ can be reduced to the Fixed Charge Transportation Problem (see [6] and [13]) and hence, it is NP-hard.

\section{Computational results}

Model $\mathscr{T}^{\mathscr{L}}$ was implemented in Python 3.6.1 using the Gurobi 7.5.1 solver, and run on an Intel Core i7-7500U $2.70 \mathrm{GHz}$ and 8GB RAM personal computer. Due to the complexity of Model $\mathscr{T}^{\mathscr{L}}$, the running is stopped when a $1 \%$ relative gap to the optimal solution or a time limit of 1 hour is reached.

We first consider the case with two retailers (i.e. $|\mathscr{I}|=2$ ). Our instances are inspired by a real case presented in [1], in which the uncertain demand of pallets should be satisfied by using trucks with limited capacity. The support of the demand probability distribution is in the set of integer numbers in the interval $[30,130]$, while the probability distribution is given by a Beta distribution $(\alpha, \beta)$, where $\alpha=20$ and $\beta=16$, having average demand $\mathbb{E}(d)=85.55556$ pallets. The supplier's inventory level $q$ is equal to 200 pallets, the capacity $C_{i}$ of the vehicles used for shipment and backordering to all retailers is equal to 34 pallets, the capacity $C^{T}$ of the vehicle used for transshipment is 17 pallets, while the retailers' warehouse capacity $Q_{i}$ is equal to 170 pallets. Furthermore, we define the value $P$ of a pallet to be equal to 1053 Euros, and since the unit inventory costs approximatively correspond to $5 \%$ of the value of a pallet of 100 kilograms, we set the supplier's inventory cost equal to $5 \% P$, and the retailers' inventory costs equal to $6 \% P$. Moreover, since the penalty cost corresponds to a lost sale and to a reputation damage, we let $p_{j}$ equal to 1.5 $P$. As in [1], we consider a unit shipment cost of a pallet with 100-200 kilograms weight on a distance up to 500 kilometers equal to 93.60 Euros and a fixed shipment cost equal to $\frac{f_{i} C_{i}}{\theta}$, where $\theta=0.5$. Finally, considering that the fixed transshipment and backordering costs are computed as a function of the unit transshipment and backordering costs, 25 different instances are generated by combining all possible values, as displayed in Table 1 . We notice that Model $\mathscr{T}^{\mathscr{L}}$ can be reduced into different special cases, which facilitate a trade-off analysis. In particular, in the "Extremely High case", obtained by assigning to transshipment and backordering costs a very high value (for example, equal to infinity), we get one instance in which both transshipment and backordering are not allowed, four instances in which only backordering is allowed and four instances in which only transshipment is allowed. The same parameters are considered also in the case with four retailers, (i.e. $|\mathscr{I}|=4$ ), apart from $q$ which is equal to 350 pallet.

In order to determine the right number of scenarios which have to be considered for the stochastic setting, we perform the in-sample stability analysis identifying as 
Table 1 Transshipment and backordering fixed and unit costs

\begin{tabular}{|c|c|c|c|c|c|}
\hline Cost & Extremely Low case (EL) & Low $(\mathrm{L})$ & Medium $(\mathrm{M})$ & High $(\mathrm{H})$ & Extremely High case $(\mathrm{EH})$ \\
\hline$t_{i j}$ & 0 & $\frac{0.75 f_{i}}{2}=35.1$ & $\frac{f_{i}}{2}=46.8$ & $\frac{1.25 f_{i}}{2}=58.5$ & $+\infty$ \\
$T_{i j}$ & 0 & $\frac{t_{i j} C^{T}}{0.5}=1193.4$ & $\frac{t_{i j} C^{T}}{0.5}=1591.2$ & $\frac{t_{i j} C^{T}}{0.5}=1989$ & $+\infty$ \\
\hline$g_{i}$ & 0 & $0.75 f_{i}=70.2$ & $f_{i}=93.6$ & $1.25 f_{i}=117$ & $+\infty$ \\
$G_{i}$ & 0 & $\frac{g_{i} C}{0.5}=4773.6$ & $F_{i}=6364.8$ & $\frac{g_{i} C}{0.5}=7956$ & $+\infty$ \\
\hline
\end{tabular}

benchmark scenario tree, the one with 500 scenarios. The out-of-sample stability analysis in the benchmark tree is obtained with 300 scenarios.

\subsection{Stochastic solution analysis}

In this section, we perform the stochastic solution analysis considering the benchmark scenario tree with 500 scenarios and computing the indicators presented in [10]. Table 2 displays the average results for the two retailers case, where with "Other" we refer to instances not belonging to any special case (i.e. the ones in which both transshipment and backordering are allowed). First, the availability of a perfect information about the future is more important if recourse decisions (i.e. backordering and transshipment) are not allowed or just transshipment is admitted with an EVPI of $12.07 \%$ in the first case and approx. $10 \%$ in the second. The case in which only backordering is allowed is the most flexible with an EVPI of $1.72 \%$, as new quantities can be introduced in the system through the recourse decision, while when only transshipment is allowed, there can be a flow of goods between retailers, but further quantities are not available. Concerning the Value of Stochastic Solution, VSS, results show there are more advantages in including stochasticity in the cases where no recourse actions are admitted or only less flexible recourse actions are allowed (i.e. transshipment). In order to understand why the deterministic solution is worse compared to the stochastic one, we compute the LUSS and the LUDS indicators. Through the LUSS, we see that in the cases where no recourse decisions or just one of them are admitted, the deterministic solution identifies the same retailers selected by the stochastic solution, but with wrong delivered quantities. In the other cases, the retailers receiving zero quantities are different in the stochastic and in the deterministic solution and, as a consequence, the poor performance is due both to the selection of retailers and to the selection of the quantities. Through the $L U D S$, we notice that the solution is perfectly upgreadable only if both backordering and transshipment are not allowed, meaning that these quantities are always lower or equal to the ones suggested by the stochastic program. For all other cases, the $L U D S$ is not null, meaning that the deterministic solution is only partially upgreadable (at least in one case, the stochastic solution delivers a lower number of pallets than the one suggested by the deterministic solution).

Finally, we focus on the case with four retailers. Due to the computational complexity of the problem, with the exception of the case "No transshipment, No back- 
Table 2 Average values for the stochastic solution analysis indicators for every special case with two retailers

\begin{tabular}{|c|c|c|c|c|c|c|c|c|c|}
\hline Cases & RP & WS & EVPI & EEV & VSS & ESSV & LUSS & EIV & LUDS \\
\hline $\begin{array}{c}\text { No transshipment } \\
\text { No backordering }\end{array}$ & 56941.68 & 50066.85 & $12.07 \%$ & 57688.54 & $1.31 \%$ & 56941.68 & $0.00 \%$ & 56941.68 & $0.00 \%$ \\
\hline Only backordering & 40856.24 & 40153.84 & $1.72 \%$ & 40956.26 & $0.25 \%$ & 40856.24 & $0.00 \%$ & 40876.56 & $0.05 \%$ \\
\hline Only transshipment & 53557.80 & 48337.35 & $9.75 \%$ & 54600.35 & $1.95 \%$ & 53557.80 & $0.00 \%$ & 53567.35 & $0.02 \%$ \\
\hline Other & 39487.43 & 38979.73 & $1.29 \%$ & 39723.29 & $0.60 \%$ & 39512.12 & $0.06 \%$ & 39504.06 & $0.04 \%$ \\
\hline
\end{tabular}

ordering", we analyze only the instances whose costs of the allowed strategy are set at a "Medium" level (i.e. only one instance for each case is considered). Results are displayed in Table 3. We specify that after 549090 seconds, the gap to the optimal solution of the $R P$ for the "Other" case was not closed and we calculate only the $E V P I$ and the VSS, since the other indicators require further constraints which make the model even more difficult to get solved to optimality. Differently from Table 2, now, if only backordering is allowed the cost is higher than the case in which only transshipment is admitted, while for the EVPI, the previous results are confirmed. Concerning the VSS, the results are now different, as there are more advantages in including stochasticity in the case where only backordering is allowed. Even if with only backordering, the quantities delivered in the first stage are fewer, transshipment is cheaper if only few quantity adjustments are needed and the presence of more retailers provides more flexibility to the distribution system.

Table 3 Values for the stochastic solution analysis indicators for every special case with four retailers and "Medium" cost level

\begin{tabular}{|c|c|c|c|c|c|}
\hline Cases & RP & WS & EVPI & EEV & VSS \\
\hline $\begin{array}{l}\text { No transshipment } \\
\text { No backordering }\end{array}$ & 120144.82 & 109191.65 & $10.03 \%$ & 131098.04 & $9.12 \%$ \\
\hline Only backordering & 112319.31 & 109191.65 & $2.86 \%$ & 122822.55 & $9.35 \%$ \\
\hline Only transshipment & 107613.10 & 103077.98 & $4.40 \%$ & 111960.39 & $4.04 \%$ \\
\hline Other & $105432.62(2.27 \%)$ & 103077.98 & $2.28 \%$ & 106535.91 & $1.05 \%$ \\
\hline
\end{tabular}

\section{Conclusions}

We presented a real problem arising in logistics and after modeling it with an integer stochastic program, we stated that this is NP-hard. Furthermore, we show that with two retailers, a decision-maker has a greater advantage by including uncertainty, especially if no recourse actions or only transshipment is admitted. We also show that in some cases, the selection of retailers to which quantities should be delivered is the same both in the deterministic and in the stochastic solution. Nevertheless, the deterministic solution can be upgrated only in the special case where no recourse 
actions are allowed. Conversely, with four retailers, transshipment provides more flexibility. Future research could be devoted to analyze the multistage version of this problem by exploiting lower bounds (see [7]-[8]) and, as in [1], to compare the stochastic solution to the one obtained through a rolling-horizon heuristic. Another stream of research could be analyzing robust optimization approaches (see [9]) or adapting approaches presented in [2].

\section{References}

1. Bertazzi, Luca and Maggioni, Francesca: A stochastic multi-stage fixed charge transportation problem: Worst-case analysis of the rolling horizon approach. European Journal of Operational Research. (2017)

2. Bertazzi, Luca and Maggioni, Francesca: Solution approaches for the stochastic capacitated traveling salesmen location problem with recourse. Journal of Optimization Theory and Applications. 166, 321-342 (2015)

3. Birge, John R and Louveaux, Francois: Introduction to stochastic programming. Springer Science \& Business Media. (2011)

4. Cavagnini, Rossana and Bertazzi, Luca and Maggioni, Francesca and Hewitt, Mike: A twostage stochastic optimization model for the Bike sharing allocation and rebalancing problem. (submitted) (2018)

5. Herer, Yale T and Rashit, Ayelet: Lateral stock transshipments in a two-location inventory system with fixed and joint replenishment costs. Naval Research Logistics (NRL). 46, 525547 (1999)

6. Klose, Andreas: Single-Sink Fixed-Charge Transportation: Applications and Exact Solution Algorithms. Working Papers, Department of Mathematical Sciences, University of Aarhus. 5 (2006)

7. Maggioni, Francesca and Allevi, Elisabetta and Bertocchi, Marida: Monotonic bounds in multistage mixed-integer stochastic programming. Computational Management Science. 13, 423457 (2016)

8. Maggioni, Francesca and Pflug, Georg: Bounds and approximations for multistage stochastic programs. Siam Journal on Optimization. 26(1), 831-855 (2016)

9. Maggioni, Francesca and Potra, Florian A and Bertocchi, Marida: A scenario-based framework for supply planning under uncertainty: stochastic programming versus robust optimization approaches. Computational Management Science. 14, 5-44 (2017)

10. Maggioni, Francesca and Wallace, Stein W: Analyzing the quality of the expected value solution in stochastic programming. Annals of Operations Research. 200, 37-54 (2012)

11. Olsson, Fredrik: An inventory model with unidirectional lateral transshipments. European Journal of Operational Research. 200, 725-732 (2010)

12. Paterson, Colin and Kiesmüller, Gudrun and Teunter, Ruud and Glazebrook, Kevin: Inventory models with lateral transshipments: A review. European Journal of Operational Research. 210, 125-136 (2011)

13. Roberti, Roberto and Bartolini, Enrico and Mingozzi, Aristide: The fixed charge transportation problem: An exact algorithm based on a new integer programming formulation. Management Science. 61, 1275-1291 (2014)

14. Rottkemper, Beate and Fischer, Kathrin and Blecken, Alexander: A transshipment model for distribution and inventory relocation under uncertainty in humanitarian operations. SocioEconomic Planning Sciences. 46, 98-109 (2012)

15. Wee, Kwan Eng and Dada, Maqbool: Optimal policies for transshipping inventory in a retail network. Management science. 51, 1519-1533 (2005)

16. Yücesan, Enver and others: Stochastic optimization for transshipment problems with positive replenishment lead times. International Journal of Production Economics. 135, 61-72 (2012) 